\title{
Immunophenotyping of serous carcinoma of the female genital tract
}

\author{
Sharon Nofech-Mozes ${ }^{1}$, Mahmoud A Khalifa ${ }^{1}$, Nadia Ismiil ${ }^{1}$, Reda S Saad ${ }^{1}$, \\ Wedad M Hanna ${ }^{1}$, Al Covens ${ }^{2}$ and Zeina Ghorab ${ }^{1}$ \\ ${ }^{1}$ Department of Anatomic Pathology, Sunnybrook Health Sciences Centre, University of Toronto, Toronto, \\ ON, Canada and ${ }^{2}$ Division of Gynecologic Oncology, Department of Obstetrics and Gynecology, \\ Sunnybrook-Odette Cancer Centre, ON, Canada
}

\begin{abstract}
To update the data on the expression of 'mesothelioma markers' by serous carcinomas of various sites we have studied cases from ovary $(n=56)$, endometrium $(n=37)$, fallopian tube $(n=6)$, primary peritoneum $(n=5)$ and cervix $(n=3)$ using a panel of antibodies (WT1, P53, estrogen receptors, HER2/neu, D2-40, cytokeratin 5/6 and E-cadherin). Ovarian carcinomas demonstrated D2-40 and cytokeratin 5/6 immunoreactivity in 23.2 and $55.4 \%$ of cases, respectively. Endometrial carcinomas demonstrated D2-40 and cytokeratin 5/6 immunoreactivity in 43.2 and $37.8 \%$ of cases, respectively. D2-40 staining pattern was predominantly focal; however, strong reactivity was identified in $16.2 \%$ of endometrial and $10.7 \%$ of ovarian carcinomas. HER2/neu oncoprotein overexpression was demonstrated in 7 of $37(18.9 \%)$ uterine serous carcinomas. In contrast, all the serous carcinomas of the other sites were HER2/neu negative. The proportion of positive cases was significantly different in ovarian vs endometrial carcinomas regarding WT1 $(P=0.0458)$, estrogen receptors $(P<0.001)$ reactivity and HER2/neu overexpression $(P=0.0025)$. D2-40 and cytokeratin $5 / 6$ are expressed in a considerable proportion of serous carcinomas and should be used cautiously in a 'mesothelioma panel' in situations where serous carcinoma is in the differential diagnosis. HER2/neu was exclusively overexpressed in serous carcinomas of endometrial origin.

Modern Pathology (2008) 21, 1147-1155; doi:10.1038/modpathol.2008.108; published online 20 June 2008
\end{abstract}

Keywords: serous carcinoma; primary peritoneal carcinoma; D2-40; CK 5/6; HER2/neu; ER

Serous carcinoma is an aggressive type of cancer that may arise from various organs of Mullerian lineage, including ovaries, endometrium, fallopian tubes, peritoneum and rarely the cervix. Serous carcinoma accounts for about half of the malignant ovarian tumors and about $10 \%$ of endometrial cancers. Despite intense investigations, ovarian serous carcinoma and uterine serous carcinoma are associated with poor prognosis. Patients with ovarian serous carcinoma most commonly present with stage III tumors and their 5-year survival is $20 \% .^{1,2}$ Uterine serous carcinoma is notorious for its propensity to invade the myometrium and lymphovascular channels and is associated with a 5-year survival of $36 \%$. This is attributable to the presence of extrauterine disease at the time of diagnosis in the majority of cases. ${ }^{1,2}$ Regardless of its origin,

Correspondence: Dr S Nofech-Mozes, MD, Department of Anatomic Pathology, Sunnybrook Health Sciences Centre, University of Toronto, 2075 Bayview Avenue E4-23a, Toronto, ON, Canada M4N 3M5.

E-mail: Sharon.nofech-mozes@sunnybrook.ca

Received 10 March 2008; revised 16 May 2008; accepted 18 May 2008; published online 20 June 2008 the tumor exhibits similar morphologic features, characterized by the presence of cellular papillae, slit-like glandular spaces, small detached buds and tufts, moderate to severe cytologic atypia, frequent mitotic activity, necrosis and occasional concentric calcification (psammoma bodies). The histologic distinction between serous carcinomas of various origins, most commonly the ovary vs endometrium, may be difficult especially in cases with advanced disease that involves more than one site in the female genital tract or when it diffusely involves the peritoneum. As uterine serous carcinoma frequently involves the ovaries, in cases with dual organ involvement it may be difficult to ascertain the primary site. Although gene expression profiling may differentiate between tumors of ovarian and endometrial origin, ${ }^{3}$ these tumors share some molecular abnormalities such as in p53 or p16. ${ }^{4}$ As a result, there is no known marker that is absolutely specific for a particular site. However, a panel of antibodies may facilitate identifying the site of origin. This panel traditionally includes WT1 and P53. ${ }^{5-8}$ Accurate identification whether a serous carcinoma originates from the ovary or the endometrium is clinically important as the pattern of tumor 
spread and the adjuvant therapy may differ, the latter is thought to exhibit a poorer response to platinum-based compounds. ${ }^{9}$ Ovarian serous carcinoma characteristically spread intraperitoneally (transcelomic); whereas uterine serous carcinoma spread by both transcelomic and lymphovascular invasion. In addition, uterine serous carcinoma commonly involves the ovaries whereas ovarian serous carcinoma rarely spread to the endometrium and is more likely to involve the uterine serosa. Immunohistochemical support for a site of origin would be helpful in cases featuring a superficially myoinvasive uterine serous carcinoma and coexistent ovarian, omental or peritoneal disease. In such cases the question arises as to whether these represent synchronous neoplasms or one tumor with a metastatic spread. Immunohistochemistry may also be useful when surgical resection is following neoadjuvant chemotherapy or when limited diagnostic material is available, ie, cell blocks from malignant ascites, biopsies of metastatic carcinoma or endometrial biopsies. Another challenge is to differentiate ovarian serous carcinoma from mesothelial cells particularly when limited diagnostic material is provided such as cytology preparation. Commonly in these cases, serous carcinoma cells should be distinguished from benign mesothelial cells that may form tight clusters that mimic ovarian tumors or less frequently from epithelioid peritoneal mesothelioma. Recently introduced immunohistochemical markers such as D2-40, CK 5/6, E-cadherin and HER2/neu (SP3) have been tested only on a limited number of cases of serous carcinoma. The proportion of positive tumors and staining pattern of these markers in serous tumors of different sites within the female genital tract is unknown. A small number of reports suggest that ovarian serous tumors express 'mesothelioma markers' ${ }^{10-12}$ D2-40, a marker of lymphatic endothelium as well as benign and malignant mesothelial cells, and a specific high molecular cytokeratin (CK) $5 / 6$ were considered as useful 'mesothelioma markers'. ${ }^{12-15}$

The aim of this study is to characterize the expression 'mesothelioma markers' and a panel of biological markers by serous tumors of the female genital tract and to explore possible variation in expression of markers among different sites.

\section{Materials and methods}

\section{Cases}

We studied 107 consecutive cases of serous carcinoma of the ovary, endometrium, fallopian tube, primary peritoneum and cervix accessioned during the period of 1999-2007 in the Department of Anatomic Pathology, Sunnybrook Health Sciences Centre in Toronto. Pertinent demographic and clinical data were retrieved from the electronic medical records.

Cases of mixed cell type were excluded from the study. Ovarian serous carcinomas were included only when destructive stromal invasion was identified. Borderline serous tumors with or without microinvasion were excluded. Ovarian serous carcinomas were graded using the Silverberg's scoring system $^{16}$ and all uterine serous carcinomas were considered as high grade. We acknowledge that neoadjuvant chemotherapy can induce morphologic changes that could potentially alter the ovarian cancer grade. The diagnosis of primary peritoneal carcinoma was made only in the absence of concurrent or previous primary endometrial, ovarian or fallopian tube carcinoma, or in the presence of microscopic foci of tumor on the external surface of the ovaries less than $5 \mathrm{~mm}$. The diagnosis of fallopian tube carcinoma was made when the fallopian tube contained the main bulk of the tumor whereas the adjacent ovary had a minor component.

The diagnosis of cervical serous carcinoma was made only in the absence of concurrent or previous primary endometrial, ovarian or peritoneal serous carcinoma as previously described. ${ }^{17}$

\section{Immunohistochemistry}

Two gynecologic pathologists reviewed the cases and selected a block containing representative tumor for immunohistochemical studies. These were performed on formalin-fixed paraffinembedded tissue sections using a panel of antibodies raised against: WT1, p53, estrogen receptors (ER), HER2/neu (SP3), D2-40, CK 5/6 and E-cadherin. The primary antibodies that were used are listed in Table 1. WT1 and p53 were included in this study mainly as

Table 1 Antibodies used in the study

\begin{tabular}{|c|c|c|c|c|}
\hline Antibody & Manufacturer & Dilution & Pretreatment & Detection \\
\hline WT1 & Dako M3561 & $1: 100$ & HIER citrate (pH 6.0) & Biocare Mach 3 Polymer \\
\hline P53 (DO7) & NovoCastra NCL-p53-DO7 & $1: 500$ & HIER citrate (pH 6.0) & Biocare Mach 3 Polymer \\
\hline $\mathrm{ER}$ & NovoCastra NCL-ER-6F11 & $1: 200$ & HIER citrate (pH 6.0) & Biocare Mach 3 Polymer \\
\hline HER2/neu (SP3) & NeoMarker RM-9103 & $1: 200$ & HIER citrate (pH 6.0) & Biocare Mach 4 Polymer \\
\hline $\mathrm{D} 2-40$ & Research lab ${ }^{a}$ & $1: 20000$ & No pretreatment & Biocare Mach 3 Polymer \\
\hline CK 5/6 & Dako M7237 & $1: 400$ & HIER Borg (pH 9.5) & Biocare Mach 3 Polymer \\
\hline E-cadherin & BD Biosciences, city and state for all of them & $1: 2000$ & HIER citrate (pH 6.0) & ZyMed LSAB detection kit \\
\hline
\end{tabular}

CK, cytokeratin; ER, estrogen receptor; lab, laboratory.

a A generous gift from Dr Marks, Women's College Hospital Toronto, Canada. ${ }^{37,38}$ 
a complementary tool in making the diagnosis of serous carcinoma. Immunostaining was recorded semiquantitatively by estimating the proportion of positive cells and classified as: negative $=0 \%$; ' $1+$ ' $=1-25 \%$; ' $2+$ ' $=25-50 \%$ and ' $3+$ ' $=$ strong ( $>50 \%$ ). HER2/neu was considered positive only when complete strong membrane staining was identified in more than $30 \%$ of the tumor, as outlined in the updated recommendations for HER2/neu testing in breast cancer. ${ }^{18}$

HER2/neu gene amplification was tested by FISH using the Vysis probe (Vysis Inc., Downers Grove, IL, USA) following the manufacturer's instructions. In brief, the, PathVysion ${ }^{\mathrm{TM}}$ kit is based on dual-color interphase analysis by using combined Her2/neu (rhodamine labeled) and chromosome $17-\alpha$-satellite DNA probes (CEP17, fluorescein labeled).

\section{Statistics}

Fisher's exact test was used to examine differences in proportions of positive stains between ovarian and endometrial carcinomas.

This study was approved by the Institutional Research Ethics Board.

\section{Results}

Immunohistochemistry was performed on 56 cases of ovarian serous carcinomas, 37 uterine serous carcinomas, 6 fallopian tube serous carcinomas, 5 primary peritoneal serous carcinomas and 3 endocervical serous carcinomas that met our inclusion criteria. The case characteristics are summarized in Table 2 and the immunohistochemical results are summarized in Table 3.

\section{WT1}

All ovarian serous carcinomas demonstrated WT1 reactivity in $>50 \%$ of the tumor cells, whereas 18 of $37(48.6 \%)$ of uterine serous carcinomas demonstrated a similar degree of reactivity for WT1
Table 3 Number of positive cases and the percentage of

\begin{tabular}{|c|c|c|c|c|}
\hline & \multicolumn{4}{|c|}{$\begin{array}{c}\text { Percentage of } \\
\text { immunoreactive cells }\end{array}$} \\
\hline & 0 & $1-25 \%$ & $25-50 \%$ & $>50 \%$ \\
\hline \multicolumn{5}{|l|}{ Ovary $(\mathrm{n}=56)$} \\
\hline WT1 & - & - & - & 56 \\
\hline P53 & 4 & 4 & 1 & 47 \\
\hline ER & 6 & 7 & 7 & 36 \\
\hline HER2/neu & 55 & - & - & - \\
\hline $\mathrm{D} 2-40$ & 43 & 7 & - & 6 \\
\hline CK 5/6 & 25 & 20 & 3 & 8 \\
\hline E-cadherin & 1 & - & - & 55 \\
\hline \multicolumn{5}{|c|}{ Endometrium $(\mathrm{n}=37)$} \\
\hline WT1 & 18 & 1 & - & 18 \\
\hline P53 & 1 & 2 & 3 & 31 \\
\hline ER & 23 & 7 & 3 & 4 \\
\hline HER2/neu & 29 & 1 & - & 7 \\
\hline $\mathrm{D} 2-40$ & 18 & 5 & 5 & 6 \\
\hline CK 5/6 & 23 & 10 & 4 & - \\
\hline E-cadherin & - & - & - & 37 \\
\hline \multicolumn{5}{|c|}{ Fallopian tube $(\mathrm{n}=6)$} \\
\hline WT1 & - & - & - & 6 \\
\hline P53 & 1 & - & - & 5 \\
\hline ER & 1 & 4 & & 1 \\
\hline HER2/neu & 6 & - & - & - \\
\hline $\mathrm{D} 2-40$ & 5 & 1 & - & - \\
\hline CK 5/6 & 6 & - & - & - \\
\hline E-cadherin & - & - & - & 7 \\
\hline \multicolumn{5}{|c|}{ Primary peritoneal $(\mathrm{n}=5)$} \\
\hline WT1 & - & 2 & 2 & 1 \\
\hline P53 & - & - & 1 & 4 \\
\hline ER & 2 & 2 & 1 & - \\
\hline HER2/neu & 5 & - & - & - \\
\hline $\mathrm{D} 2-40$ & 5 & - & - & - \\
\hline CK $5 / 6$ & 5 & - & - & - \\
\hline E-cadherin & - & - & - & 5 \\
\hline \multicolumn{5}{|l|}{ Cervix $(\mathrm{n}=3)$} \\
\hline WT1 & 2 & - & - & 1 \\
\hline P53 & 1 & - & - & 2 \\
\hline ER & 2 & 1 & - & - \\
\hline HER2/neu & 3 & - & - & - \\
\hline $\mathrm{D} 2-40$ & 5 & - & - & - \\
\hline CK 5/6 & - & - & - & 3 \\
\hline E-cadherin & - & - & - & 3 \\
\hline
\end{tabular}

CK, cytokeratin; ER, estrogen receptor.

Table 2 Case characteristics

\begin{tabular}{|c|c|c|c|c|c|}
\hline Site of origin & $\begin{array}{c}\text { Ovary } \\
(\mathrm{n}=56)\end{array}$ & $\begin{array}{l}\text { Endometrium } \\
\quad(\mathrm{n}=37)\end{array}$ & $\begin{array}{l}\text { Fallopian tube } \\
\qquad(\mathrm{n}=6)\end{array}$ & $\begin{array}{l}\text { Primary peritoneal } \\
\qquad(\mathrm{n}=5)\end{array}$ & $\begin{array}{c}\text { Cervical } \\
(\mathrm{n}=3)\end{array}$ \\
\hline Age (years) & $59.4 \pm 13.5$ & $71.1 \pm 8.1$ & $56.1 \pm 4.1$ & $70.1 \pm 9.7$ & $58.5 \pm 6.4$ \\
\hline Grade 1 & 9 & - & 1 & 1 & - \\
\hline Grade 2 & 7 & - & - & 1 & - \\
\hline Grade 3 & 35 & 37 & 5 & 3 & 3 \\
\hline Unspecified $^{\mathrm{a}}$ & 5 & - & - & - & - \\
\hline Neoadjuvant chemotherapy & 30 & - & 2 & - & - \\
\hline
\end{tabular}

${ }^{\mathrm{a}}$ Neoadjuvant chemotherapy-related changes preclude accurate grading. 
$(P=0.0458)$. In all fallopian tube carcinomas, 1 of 5 $(20 \%)$ primary peritoneal carcinomas and 1 of 3 cervical serous carcinomas $>50 \%$ of the tumor were WT1 immunoreactive.

\section{P53}

The proportion of strong p53 reactivity was comparable in all disease sites. p53 was demonstrated in more $>50 \%$ of the tumor in 47 of $56(83.6 \%)$ ovarian serous carcinomas, 28 of $34(83.8 \%)$ uterine serous carcinomas, 5 of $6(83.9 \%)$ fallopian tube, 4 of 5 $(80 \%)$ primary peritoneal carcinomas and in 2 of 3 cervical serous carcinomas.

\section{Estrogen Receptors}

Estrogen receptor reactivity was demonstrated in more than $50 \%$ of the tumor of 36 of $56(64.3 \%)$ ovarian serous carcinomas, whereas most of the uterine serous carcinomas were ER negative. Only 4 of $37(10.8 \%)$ demonstrated ER reactivity in more than $50 \%$ of the tumor $(P<0.001)$. Similar degree of ER reactivity was demonstrated only in 1 of $6(16.7 \%)$ fallopian tube tumors and in none of the primary peritoneal or cervix serous carcinomas.

\section{HER2/neu}

Strong complete membranous stain consistent with HER2/neu oncoprotein overexpression was demonstrated in 7 of $37(18.9 \%)$ uterine serous carcinomas (Figure 1). Notably, one additional uterine serous carcinoma was only focally HER2/neu positive. This case was further studied by FISH that showed focal HER2/neu gene amplification. The FISH score in the IHC-positive area was 3 whereas that of the IHCnegative area was 1 . None of the other sites including ovary, fallopian tubes, primary peritoneal
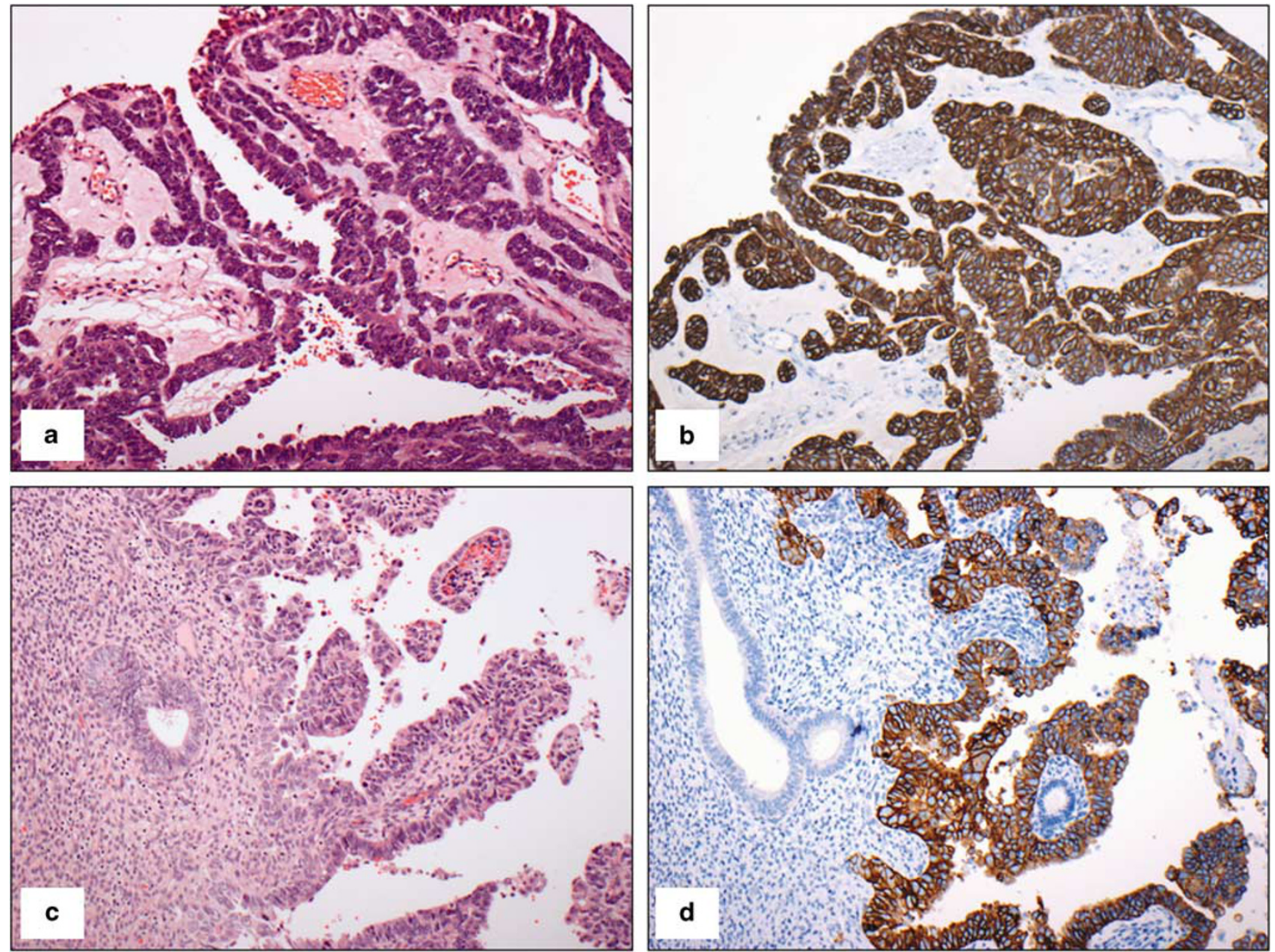

Figure 1 (a) Hematoxylin-eosin, uterine serous carcinoma. (b) HER2/neu oncoprotein (SP3) is overexpressed. Note complete strong membranous staining. (c) Hematoxylin-eosin, uterine serous carcinoma and adjacent benign endometrial gland. (d) HER2/neu oncoprotein (SP3) is overexpressed in the tumor, the benign endometrial gland is negative. 

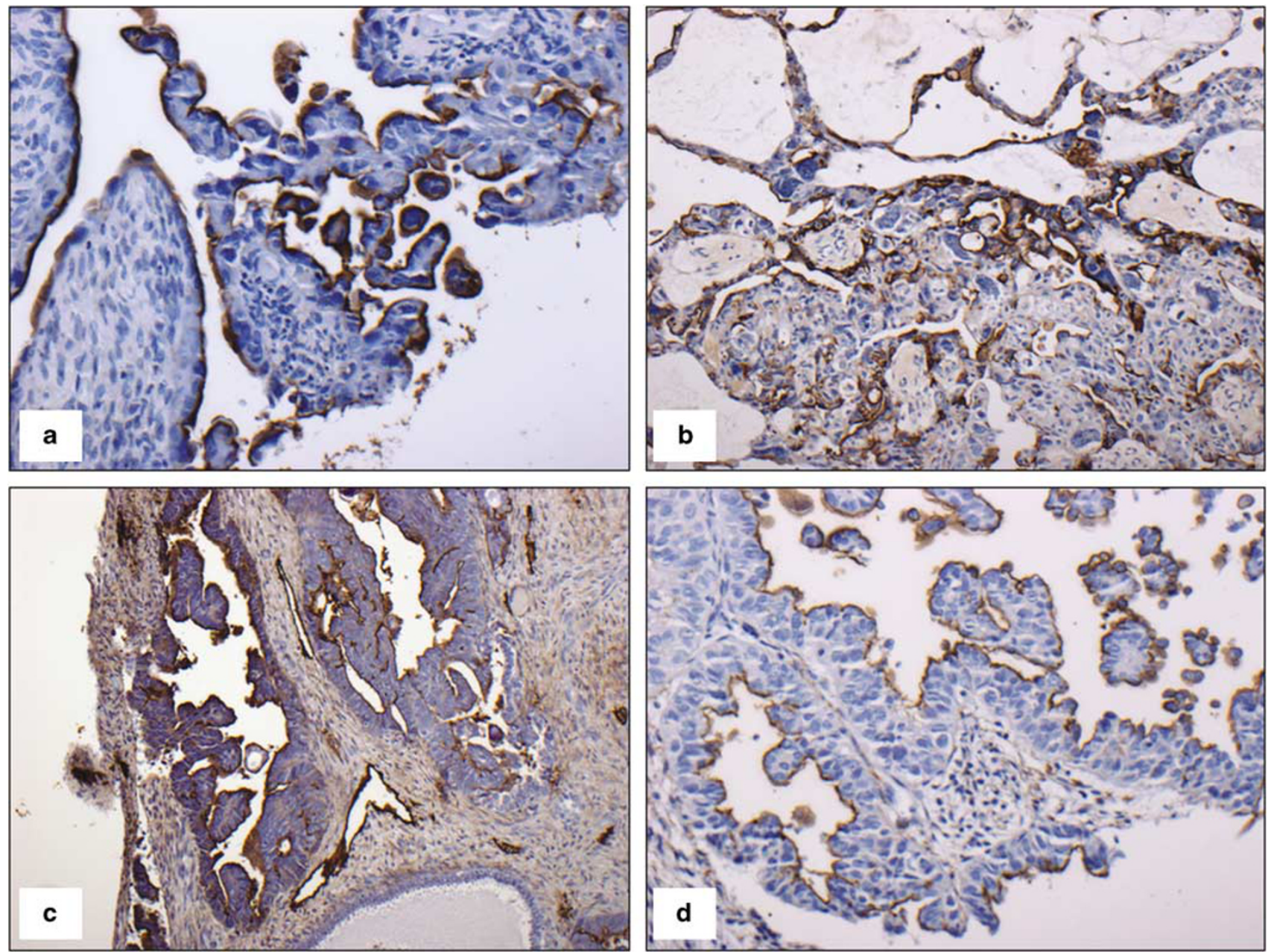

Figure 2 Ovarian serous carcinoma: D2-40 immunohistochemistry. (a) Positive staining along the apical cell membrane. (b) Patient treated with neoadjuvant chemotherapy with similar staining pattern. Uterine serous carcinoma: D2-40 immunohistochemistry. (c) Continuous-positive staining along the apical cell membrane. Note D2-40 is also positive in stromal lymphatics. The benign adjacent endometrial gland is negative. (d) Uterine serous carcinoma, similar staining pattern.

and cervix tumors overexpressed HER2/neu oncoprotein. The difference in the proportion of positive cases between endometrial and ovarian tumors was significant $(P=0.0025)$.

\section{D2-40}

Some degree of D2-40 reactivity was demonstrated in 13 of $56(23.2 \%)$ ovarian serous carcinomas, but only in 6 of $56(10.7 \%)$ cases $>50 \%$ of the tumor was positive (Figure 2a and b). Some degree of D2-40 reactivity was seen in 16 of $37(43.2 \%)$ uterine serous carcinomas but only in 6 of $37(16.2 \%)$ cases $>50 \%$ of the tumor was positive (Figure 2c and d). The difference between D2-40 expression in ovarian vs endometrial tumors was not statistically significant $(P=0.198)$. Positive cases from other sites demonstrated less than $50 \%$ of the tumor cells were immunoreactive. Characteristically, the staining pattern was along the apical cell membrane though occasional cases exhibited continuous membranous stain encircling the cells.

\section{CK 5/6}

Some degree of CK 5/6 reactivity was demonstrated in 31 of $56(55.4 \%)$ ovarian serous carcinomas but $>50 \%$ of the tumor was reactive only in 8 of 56 (14.3\%; Figure 3). Some degree of CK 5/6 reactivity was demonstrated in 14 of 37 (37.8\%) uterine serous carcinomas (Figure 4). None of the uterine serous carcinomas, fallopian tube carcinomas and primary peritoneal carcinomas reacted with CK $5 / 6$ in $>50 \%$ of the tumor. The three cases of cervical serous carcinoma demonstrated CK 5/6 reactivity in $>50 \%$ of the tumor.

The difference between CK 5/6 expression in ovarian vs endometrial tumors was not statistically significant $(P=0.349)$. In ovarian and uterine serous carcinomas that were focally reactive for both 

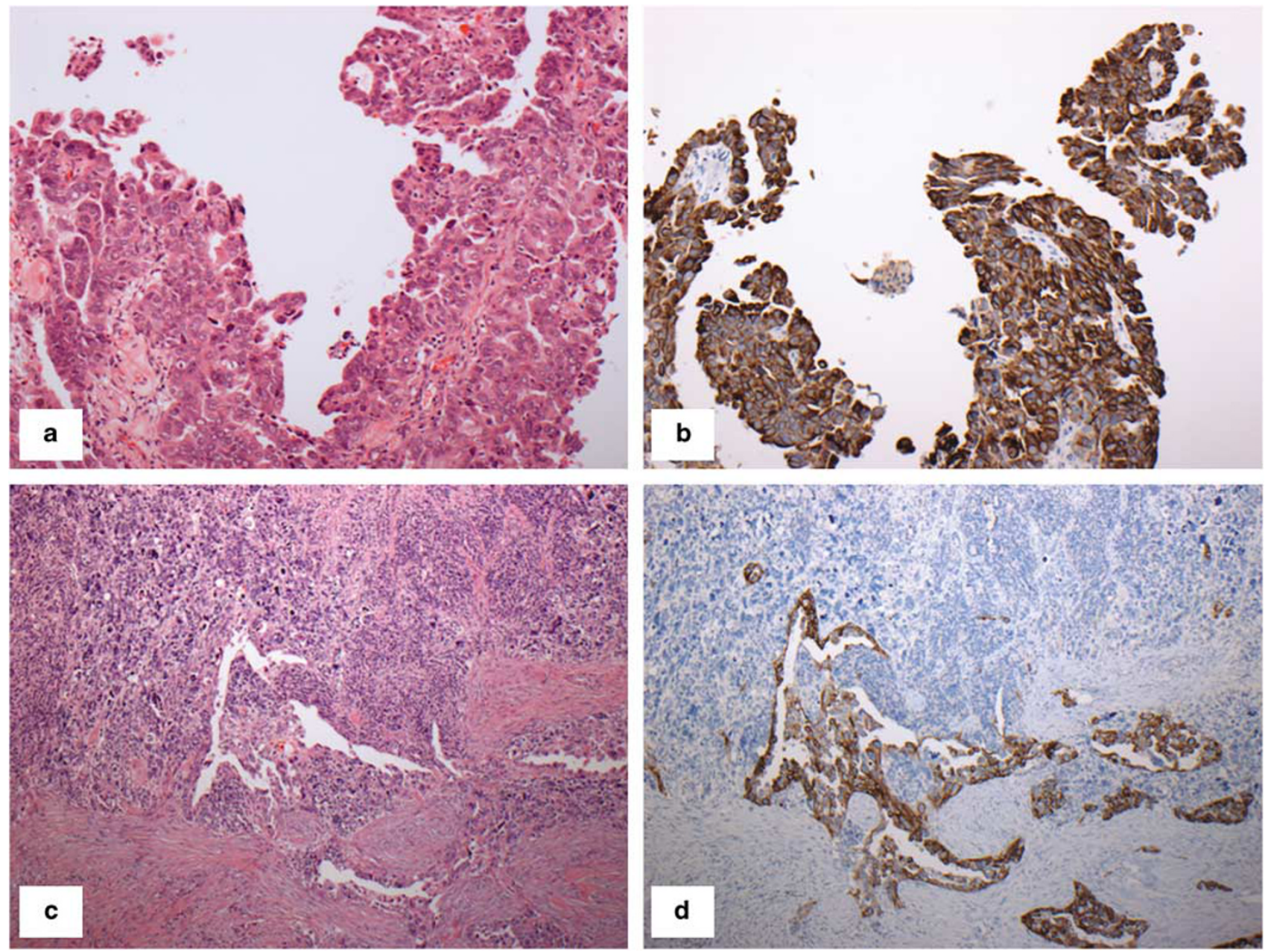

Figure 3 (a) Ovarian serous carcinoma; hematoxylin-eosin. (b) A representative case of ovarian serous carcinoma with strong and diffuse CK 5/6 immunoreaction. (c) Ovarian serous carcinoma; hematoxylin-eosin. (d) A representative case of ovarian serous carcinoma with CK $5 / 6$ immunoreaction in less than $25 \%$ of the tumor cells. This was the predominant pattern.
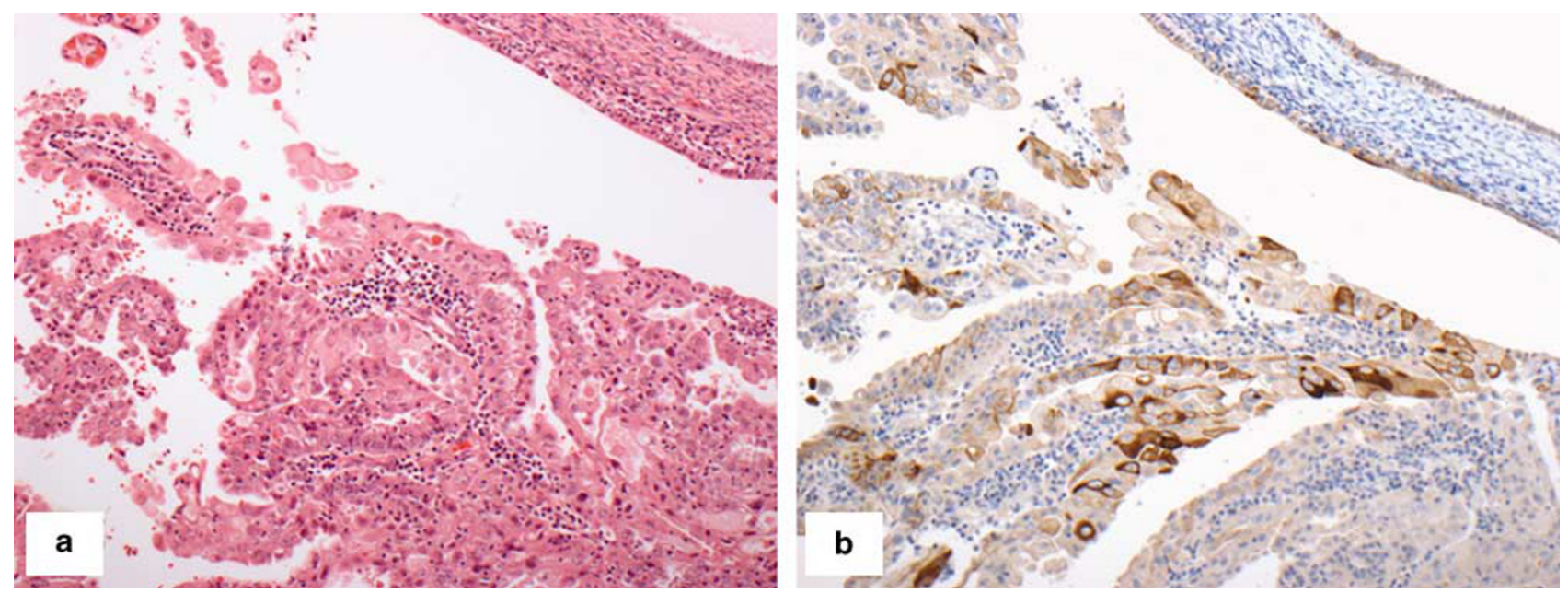

Figure 4 (a) Uterine serous carcinoma; hematoxylin-eosin. (b) CK 5/6-positive immunoreaction is demonstrated in less than $25 \%$ of the tumor cells. 
CK 5/6 and D2-40, the subset of CK 5/6-positive cells did not overlap with D2-40-positive cells.

\section{E-cadherin}

Regardless of their site of origin, almost all serous carcinomas that were included in the study exhibited diffuse E-cadherin reactivity. One ovarian serous carcinoma was negative in repeated staining with a positive internal control.

\section{Effect of Neoadjuvant Therapy}

In 30 of $56(53.6 \%)$ of the ovarian serous carcinoma patients, neoadjuvant chemotherapy was administered prior to the primary surgical treatment. There was no significant association between neoadjuvant therapy and expression of any of the markers.

\section{Association with Tumor Grade}

When low grade (Silverberg's grade 1) was compared with high-grade (Silverberg's grades 2 and 3 ) ovarian serous carcinomas, there was no significant association between the ovarian tumor grade and the expression of any of the markers.

\section{Discussion}

D2-40 antibody reacts with a mucin-type transmembrane sialoglycoprotein ${ }^{19,20}$ expressed in lymphatic endothelium, seminoma, benign and malignant mesothelial cells. ${ }^{15,20,21}$ In the female genital tract D2-40 is expressed in germ cell neoplasia, sex cord stromal tumors and adenomatoid tumors. ${ }^{19,22}$ Previous studies suggested that D2-40 and CK 5/6 are specific to mesothelium and recommended their use to differentiate mesothelioma from serous carcinoma of the ovary. ${ }^{13,23,24}$ In our study, D2-40 and CK 5/6 expression was demonstrated in a substantial number of serous carcinoma of ovarian (23.2 and $55.4 \%$, respectively) and endometrial origin (43.2 and $37.8 \%$, respectively). Nevertheless, unlike the diffuse reactivity characteristic to mesothelioma, the expression of D2-40 and/or CK 5/6 in serous carcinomas was usually confined to a subset of the neoplastic cells; typically the stain was positive in less than $25 \%$ of the cells. Our findings confirm results of a previous study that demonstrated low specificity for D2-40 as a mesothelial marker, especially in the context of differentiating mesothelial cells from an ovarian carcinoma. Unlike our study, the aforementioned study examined D2-40 expression in effusion specimens and in multitissue microarray that included only 23 cases of primary or metastatic unspecified ovarian carcinomas. The authors reported that $19 \%$ of the cores and more than $50 \%$ of effusions of ovarian carcinomas were positive for D2-40. ${ }^{11}$ Ordóñez et al compared the immunohistochemical profile of ovarian serous carcinoma with epithelioid mesotheliomas using a panel of 15 markers. In their study, none of the ovarian serous carcinoma reacted strongly with D2-40 and CK 5/6; however, $13 \%$ exhibited focal D2-40 reactivity. Chu et al noted that 17 of 26 (65\%) of the ovarian serous carcinoma included in their study, exhibited focal D2-40 reactivity. The difference in proportion of D2-40 and CK 5/6-positive cases was not significant when ovarian serous carcinoma were compared with uterine serous carcinoma in our study. Ionescu et $a l^{25}$ suggested that CK 5/6 expression is significantly higher in uterine serous carcinoma based on a TMA study. They found that CK $5 / 6$ was expressed in $48 \%$ of uterine serous carcinoma (compared to $37.8 \%$ in our study) but only in $5 \%$ of ovarian serous carcinoma (compared to $56.4 \%$ in our study). Their observation regarding HER2/neu oncoprotein overexpression in uterine serous carcinoma is in line with our findings; however, their cut off for positivity was not specified.

As shown by an oligonucleotide microarray study assessing the differential expression of approximately 10000 genes between ovarian serous carcinoma and uterine serous carcinoma, HER-2/neu is the most strikingly overexpressed gene in uterine serous papillary carcinoma compared with serous epithelial ovarian cancer. ${ }^{26,27}$ It has been shown that HER-2/neu oncoprotein overexpression is significantly associated with poor survival in uterine serous carcinoma ${ }^{28}$ and could also be important as a predictive factor in uterine serous carcinoma treatment. It has been related to resistance to chemotherapy and poor prognosis in patients with this aggressive uterine malignancy. ${ }^{29}$ Trastuzumab, a humanized monoclonal antibody specific for HER-2/ neu, is important in the adjuvant and metastatic treatment of patients with HER-2/neu-overexpressing breast cancer. HER-2/neu overexpression in uterine serous carcinoma provides a biological rationale for the use of trastuzumab in the treatment of this malignancy. ${ }^{27,30,31} \mathrm{~A}$ novel rabbit monoclonal antibody directed against HER2/neu oncoprotein SP3 was recently introduced and is used in routine clinical diagnosis in our laboratory. We have shown that using this novel antibody for HER2/neu testing reduces the rate of equivocal immunohistochemical results and highly correlates with HER2/neu gene amplification. ${ }^{32}$ In endometrial cancer, HER2/neu oncoprotein overexpression has been associated with type II endometrial cancer, namely uterine serous carcinoma. An earlier study ${ }^{26}$ on HER2/neu status in uterine serous carcinoma reported that $35 \%$ of 26 cases examined overexpressed HER2/neu oncoprotein and demonstrated gene amplification in all the cases scored $3+$ by immunohistochemistry. Interestingly, they also noted cases with intratumoral heterogeneity.

Our observation that ER is expressed in almost all $(89 \%)$ ovarian serous carcinomas is in line with previous studies. Ordóñez ${ }^{33}$ reported ER expression 
in $88 \%$ of ovarian serous carcinomas $(n=40)$, with similar frequency in primary peritoneal carcinomas, and Comin et $a l^{34}$ found that $95 \%$ of ovarian serous carcinomas included in their study were ER positive. The expression of ER in ovarian serous carcinomas in our study was not associated with tumor grade or neoadjuvant chemotherapy. In contrast to our observation, higher ER and E-cadherin expression was reported for low-grade ovarian serous carcinomas than for high-grade ovarian serous carcinomas by Wong et al. ${ }^{35}$ It is possible that we could not detect a significant difference in ER expression between low- and high-grade ovarian serous carcinoma in any of the markers results from limited sample size of low-grade tumors in our study. O'Neill et $a l^{36}$ found significantly higher expression of p53 and HER2/neu in high-grade ovarian serous carcinoma when compared with low-grade tumors. The authors used a different cut off in their statistical analysis as well as a different antibody for HER2/neu testing that may account for the difference between our findings and their report.

In accordance with our observation, expression of WT1 by serous carcinoma other then ovarian origin has been previously demonstrated in fallopian tube and primary peritoneal but not in uterine serous carcinomas. ${ }^{8}$ Acs et $a l^{7}$ found that a subset of uterine serous carcinoma (10 of 16) express WT1; however, the level of WT1 expression in their study was significantly different among serous carcinomas arising at different sites $(P<0.0001)$. Similar to our findings the authors did not find a significant difference in p53 expression among the different sites.

In summary, using a panel of seven antibodies, we found a significant difference in immunoreactivity of ovarian and endometrial carcinomas regarding WT1 $(P=0.0458)$ and ER $(P<0.001)$ reactivity as well as HER2/neu oncoprotein overexpression $(P=0.0025)$. Strong WT1 and ER expression were associated with ovarian primary whereas HER2/neu oncoprotein overexpression was associated with endometrial origin.

D2-40 and CK 5/6 are expressed in considerable proportion of serous carcinomas and should be used cautiously in a 'mesothelioma panel' in particular when used to differentiate benign reactive mesothelial proliferation or epithelioid mesothelioma from serous carcinoma in fluid cytology. The focal CK 5/6 and D2-40 expression in substantial number of cases may limit the value of microarray studies. HER2/neu was exclusively overexpressed in serous carcinomas of endometrial origin and although expressed in limited subset of cases, could be used to identify endometrial origin in the context of advanced serous carcinomas, and may be used to select patients for targeted therapy.

\section{Acknowledgement}

We thank Kevin Kowk for his skillful technical assistance. This study was presented in part in the
United States and Canadian Academy of Pathology Annual Meeting, Denver, Colorado, March 2008.

\section{References}

1 Crum CP, Lee KR. The pathology of surface epithelialstromal tumors of the ovary. Diagnostic Gynecologic and Obstetric Pathology. Elsevier Saunders: Philadelphia, PA, USA, 2006, pp 839-903.

2 Kurman RJ. Blaustein's Pathology of the Female Genital Tract, 5th edn. Springer-Verlag: Baltimore, MD, 2001.

3 Zorn KK, Bonome T, Gangi L, et al. Gene expression profiles of serous, endometrioid, and clear cell subtypes of ovarian and endometrial cancer. Clin Cancer Res 2005;11:6422-6430.

4 Chiesa-Vottero AG, Malpica A, Deavers MT, et al. Immunohistochemical overexpression of p16 and p53 in uterine serous carcinoma and ovarian high-grade serous carcinoma. Int J Gynecol Pathol 2007;26: 328-333.

5 Al-Hussaini M, Stockman A, Foster H, et al. WT-1 assists in distinguishing ovarian from uterine serous carcinoma and in distinguishing between serous and endometrioid ovarian carcinoma. Histopathology 2004;44:109-115.

6 Goldstein NS, Uzieblo A. WT1 immunoreactivity in uterine papillary serous carcinomas is different from ovarian serous carcinomas. Am J Clin Pathol 2002;117: 541-545.

7 Acs G, Pasha T, Zhang PJ. WT1 is differentially expressed in serous, endometrioid, clear cell, and mucinous carcinomas of the peritoneum, fallopian tube, ovary, and endometrium. Int J Gynecol Pathol 2004;23:110-118.

8 Hashi A, Yuminamochi T, Murata S, et al. Wilms tumor gene immunoreactivity in primary serous carcinomas of the fallopian tube, ovary, endometrium, and peritoneum. Int J Gynecol Pathol 2003;22:374-377.

9 Resnik E, Taxy JB. Neoadjuvant chemotherapy in uterine papillary serous carcinoma. Gynecol Oncol 1996;62:123-127.

10 Jones DL, Denning KL, Saad RS, et al. Diagnostic limitation of D2-40 in separating serous carcinoma from other metastatic carcinoma of the abdomen [abstract]. Mod Pathol 2006;19:183A.

11 Bassarova AV, Nesland JM, Davidson B. D2-40 is not a specific marker for cells of mesothelial origin in serous effusions. Am J Surg Pathol 2006;30:878-882.

12 Chu AY, Litzky LA, Pasha TL, et al. Utility of D2-40, a novel mesothelial marker, in the diagnosis of malignant mesothelioma. Mod Pathol 2005;18:105-110.

13 Ordóñez NG. The diagnostic utility of immunohistochemistry and electron microscopy in distinguishing between peritoneal mesotheliomas and serous carcinomas: a comparative study. Mod Pathol 2006;19: 34-48.

14 Ordóñez NG. D2-40 and podoplanin are highly specific and sensitive immunohistochemical markers of epithelioid malignant mesothelioma. Hum Pathol 2005;36: 372-380.

15 Bhalla R, Siddiqui MT, Mandich D, et al. Diagnostic utility of D2-40 and podoplanin in effusion cell blocks. Diagn Cytopathol 2007;35:342-347. 
16 Silverberg SG. Histopathologic grading of ovarian carcinoma: a review and proposal. Int J Gynecol Pathol 2000;19:7-15.

17 Nofech-Mozes S, Rasty G, Ismiil N, et al. Immunohistochemical characterization of endocervical papillary serous carcinoma. Int J Gynecol Cancer 2006;16: 286-292.

18 Wolff AC, Hammond ME, Schwartz JN, et al. American Society of Clinical Oncology/College of American Pathologists guideline recommendations for human epidermal growth factor receptor 2 testing in breast cancer. J Clin Oncol 20071;25:118-145.

19 Schacht V, Dadras SS, Johnson LA, et al. Up-regulation of the lymphatic marker podoplanin, a mucin-type transmembrane glycoprotein, in human squamous cell carcinomas and germ cell tumors. Am J Pathol 2005;166:913-921.

20 Kahn HJ, Bailey D, Marks A. Monoclonal antibody D240, a new marker of lymphatic endothelium, reacts with Kaposi's sarcoma and a subset of angiosarcomas. Mod Pathol 2002;15:434-440.

21 Ordóñez NG. Podoplanin: a novel diagnostic immunohistochemical marker. Adv Anat Pathol 2006; 13:83-88.

22 Ghorab Z, Khalifa MA, Kahn HJ. Adenomatoid tumors of female and male genital tract express D2-40 [abstract]. Mod Pathol 2006;19:138A.

23 Hanley KZ, Facik MS, Bourne PA, et al. Utility of antiL523S antibody in the diagnosis of benign and malignant serous effusions. Cancer 2008;114:49-56.

24 Cury PM, Butcher DN, Fisher C, et al. Value of the mesothelium-associated antibodies thrombomodulin, cytokeratin 5/6, calretinin, and $\mathrm{CD} 44 \mathrm{H}$ in distinguishing epithelioid pleural mesothelioma from adenocarcinoma metastatic to the pleura. Mod Pathol 2000;13:107-112.

25 Ionescu DN, Chebib I, Alkushi A, et al. Immunohistochemical comparison of serous carcinomas of ovary vs endometrium [abstract]. Mod Pathol 2007;20:201A.

26 Santin AD, Bellone S, Van SS, et al. Determination of HER2/neu status in uterine serous papillary carcinoma: comparative analysis of immunohistochemistry and fluorescence in situ hybridization. Gynecol Oncol 2005;98:24-30.

27 Santin AD, Zhan F, Cane' S, et al. Gene expression fingerprint of uterine serous papillary carcinoma: identification of novel molecular markers for uterine serous cancer diagnosis and therapy. Br J Cancer 2005; 92:1561-1573.
28 Santin AD, Bellone S, Van SS, et al. Amplification of cerbB2 oncogene: a major prognostic indicator in uterine serous papillary carcinoma. Cancer 2005; 104:1391-1397.

29 Gadducci A, Cionini L, Romanini A, et al. Old and new perspectives in the management of high-risk, locally advanced or recurrent, and metastatic vulvar cancer. Crit Rev Oncol Hematol 2006; 60:227-241.

30 Slomovitz BM, Ramondetta LM, Lee CM, et al. Heterogeneity of stage IIIA endometrial carcinomas: implications for adjuvant therapy. Int J Gynecol Cancer 2005;15:510-516.

31 Santin AD, Bellone S, O’Brien TJ, et al. Current treatment options for endometrial cancer. Expert Rev Anticancer Ther 2004:4:679-689.

32 Ghorab Z, Kahn H, Hanna WM. Her 2/neu status in invasive breast cancers assessed by SP3: correlation with TAB250 and FISH [abstract]. Mod Pathol 2007;20:348A.

33 Ordóñez NG. Value of estrogen and progesterone receptor immunostaining in distinguishing between peritoneal mesotheliomas and serous carcinomas. Hum Pathol 2005;36:1163-1167.

34 Comin CE, Saieva C, Messerini L. h-caldesmon, calretinin, estrogen receptor, and Ber-EP4: a useful combination of immunohistochemical markers for differentiating epithelioid peritoneal mesothelioma from serous papillary carcinoma of the ovary. Am J Surg Pathol 2007;31:1139-1148.

35 Wong KK, Lu KH, Malpica A, et al. Significantly greater expression of ER, PR, and ECAD in advancedstage low-grade ovarian serous carcinoma as revealed by immunohistochemical analysis. Int J Gynecol Pathol 2007;26:404-409.

36 O’Neill CJ, Deavers MT, Malpica A, et al. An immunohistochemical comparison between low-grade and high-grade ovarian serous carcinomas: significantly higher expression of p53, MIB1, BCL2, HER-2/ neu, and C-KIT in high-grade neoplasms. Am J Surg Pathol 2005;29:1034-1041.

37 Kahn HJ, Marks A. A new monoclonal antibody, D2-40, for detection of lymphatic invasion in primary tumors. Lab Invest 2002;82:1255-1257.

38 Marks A, Sutherland DR, Bailey D, et al. Characterization and distribution of an oncofetal antigen (M2A antigen) expressed on testicular germ cell tumours. Br J Cancer 1999;8:569-578. 